\title{
Bayesian Network Model of Product Information Diffusion and Reasoning of Influence
}

\author{
Xuehua Sun $^{1 *}$ (D), Shaojie Hou${ }^{1}$, Ning Cai' ${ }^{2}$, Wenxiu Ma1 ${ }^{1}$, Surui Zhao ${ }^{1}$ \\ ${ }^{1}$ School of Information Technology, Hebei University of Economics and Business, Shijiazhuang, China \\ ${ }^{2}$ School of Culture and Communication, Hebei University of Economics and Business, Shijiazhuang, China \\ Email: *sunxuehua795@126.com
}

How to cite this paper: Sun, X.H., Hou, S.J., Cai, N., Ma, W.X. and Zhao, S.R. (2020) Bayesian Network Model of Product Information Diffusion and Reasoning of Influence. Journal of Data Analysis and Information Processing, 8, 267-281. https://doi.org/10.4236/jdaip.2020.84015

Received: September 14, 2020

Accepted: October 27, 2020

Published: October 30, 2020

Copyright () 2020 by author(s) and Scientific Research Publishing Inc. This work is licensed under the Creative Commons Attribution International License (CC BY 4.0).

http://creativecommons.org/licenses/by/4.0/

(c) (i) Open Access

\begin{abstract}
Information diffusion on social media has become a key strategy in people's daily interactions. This paper studies consumers' participation in the product information diffusion, and analyzes the complexity of information diffusion which is affected by many factors. Prior investigations of information diffusion have primarily focused on the composition of diffusion networks with independent factors and the intricacy of the process has not been completely evaluated. The majority of prior investigations have focused on strategies and the moving forces in social media processes and the determination of influential seed nodes, with few evaluations conducted about the factors affecting consumers' choices in information diffusion. In this study, a Bayesian network model of product information diffusion was created to examine the links between factors and consumer deportment. It revealed how those factors had an impact on each other and on consumer deportment choice. The innovation of the thesis is reflected in the exploration and analysis of the specific communication path of product information diffusion, which provides a better marketing idea and practical method for the development of mobile e-commerce. The research findings can help identify the quantitative relationships between the factors affecting the process of product information diffusion and user behavior.
\end{abstract}

\section{Keywords}

Product Information Diffusion, Bayesian Network Model, Influence Reasoning, Consumer Behaviors, Clique Tree

\section{Introduction}

Consumers generate a large quantity of data online and naturally create their in- 
dividual marketing networks as consumer-led media and technology-including mobile intelligent terminals, WeChat, and social media sites-quickly grow, obscuring the boundaries between industries and consumers. Consumers (as content creators and data publishers) have established their unique media networks, while enterprises publish a vast quantity of product information via numerous forms of social media-including network platforms and intelligent media terminals-to gain the attention of possible users. Enticements have been offered to prompt consumers to reveal product information on their self-organized social media so that consumers are converted from individuals who peruse product information and buy products into product advocates and enterprise partners. These types of associations are advantageous for both enterprises and consumers.

Consumers have a unique group of friends on social media, and since consumers are faced with vast quantities of data and media advertising and therefore are not always certain how to make the correct selection, they trust their friends' recommendations (Figure 1).

Information cascade is the main method of information diffusion. In social media, people often forward information posted by someone else, with such information usually coming from the user's neighbors (friends) in the social network. The spread of information between friends leads to information cascade. The most representative information diffusion models are discussed below. Kempe et al. [1] proposed two equivalent models: the general cascade (GC) model and the general threshold (GT) model, which are generalizations of the independent cascade (IC) model and the linear threshold (LT) model. In the GC model, the probability of node diffusion depends on the record of historical activation, while this probability is set as a constant in the IC model. In the GT model, the threshold function determining whether each node is activated is the function of the weight of activated nodes; however, in the LT model, it is the sum of the weight of all of the activated nodes. In the IC and LT models, each activated node attempts to influence the nodes that have not been activated, and in the voter model [2], there are two options for each node, with two kinds of information competing to activate more nodes.

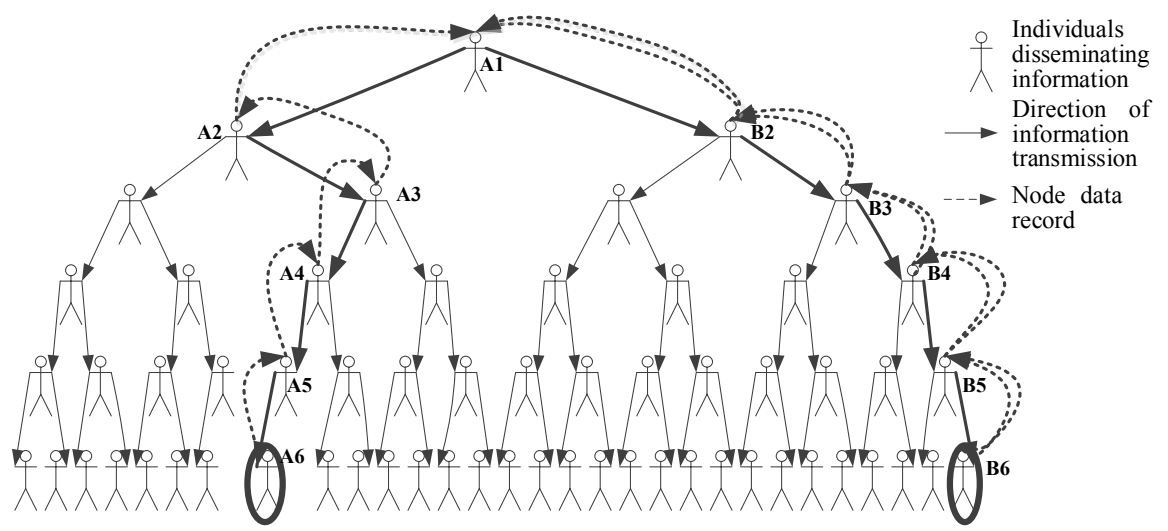

Figure 1. Product information allocation and value generation in networks self-organized by consumers. 
In addition to the traditional information diffusion models mentioned above, there are also many expansion models based on these models. The IC model with negative opinions (IC-N) [3] takes into account the diffusion of negative information. In the IC-N model, if a positive activated node successfully activates the silent nodes, then positive and negative information will be spread at the same time; if a negative activated node successfully activates the silent nodes, then only negative information will be diffused. Extending the LT model, the Competitive LT model (CLT) model [4] considers two kinds of competing information in the network; in the CLT model, the seed nodes are activated and attach to one of the two competing types of information to be spread; in the process of activating the silent nodes, the seed nodes try to persuade the silent nodes to accept the information they supported. The Signed Voter model [5] extended the Voter Model. When the two nodes on one edge were friends, one node successfully activated the other node and attempted to persuade it to accept the information he or she supported. When successfully activating the silent nodes, the IC-N model and the CLT model produce similar negative impacts, namely, making the other node hold the same opinion.

The information diffusion model explored in this study is different from previous models in the following aspects:

1) A different problem was studied. Most currently available models consider 1 - 2 factors of information diffusion at most, and they tend to lock information sources and identify the seed nodes [6] [7] [8] [9] [10], but it remains unknown how the product information transmission chain initiated by the seed nodes can influence consumer behavior. Therefore, in this study, multiple factors affecting the process of information diffusion were extracted, the degree to which they influence consumer behavior choice was analyzed, and the quantitative relationship between the two was explored.

2) Different methods are used. This study adopted Bayesian network modeling, giving it two major advantages: a) it is multi-factor and b) due to conditional independent distribution and the chain rule of the Bayesian network, the number of parameters in the table is exponentially smaller than the number of parameters in joint distribution, that is, the conditional probability distribution (CPD) of the nodes in the Bayesian network depends only on the father nodes. Even if $n$, the node of the entire Bayesian network, is very large, $k$, the father node of each node is very small, reducing the complexity of the operation, which is a major advantage of the Bayesian network.

The logic structure of the study is as follows: in the subsequent section, the product information diffusion Bayesian network model is established. In the third part, the influence reasoning process is explored. In the fourth part, the reasoning process is analyzed and the influence reasoning is verified. The conclusions are presented in the fifth part.

\section{Product Information Diffusion Bayesian Network Model}

As a probabilistic digraph, a Bayesian network can naturally express people's 
causal intelligence with digraphs, perform multifactorial modeling, graphically demonstrate joint probability distribution among random variables, and handle numerous undetermined factors.

In this study, multiple factors during the information diffusion process should be taken into consideration to model product information diffusion; this is an advantage of using the Bayesian network to express data with such structures. Thus, a Bayesian network was implemented in this study to demonstrate the data organization of the product information diffusion network.

\subsection{Establishment of Random Variables in the Product Information Diffusion Process}

Variables were chosen for implementation in the Bayesian network modeling of the information diffusion process according to three features: network structure, information attributes, and user attributes. They were defined as:

1) Network structure

Cross diffusion: Cross diffusion encourages information diffusion in numerous groups through the elimination of clustering inside the community structure of social media that prevents large-scale diffusion of information.

2) Information attributes

Theme: Product information intently developed by enterprises is diffused on social media, while social media users display messages regarding certain products to create a theme.

Emotion conveyed by each piece of information: this covers disagreements over a particular theme or idea, including both positive and negative information.

Origin of information: Social media generate a vast amount of real-time content at an outstanding pace, and associations between users can impact users' discernment of information. Concurrently, the value of the information will impact associations among users. Thus, the origin of the information is critical for information diffusion.

Resonance of users toward the information: The manner in which emotions conveyed by information settles with users is of particular significance for information forwarding.

3) User attributes

User state: This involves seed nodes, diffusion nodes, and information nodes. Seed nodes possess a vast quantity of connections that impact the diffusion of information, and they are typically efficient information origins in product information diffusion. Their greatest function is to alert consumers to novel products and stimulate large information cascades. Diffusion nodes compel users' forwarding behaviors as well as the information cascades stimulated by the seed nodes. It is usually challenging for information nodes to initiate lengthy chain responses, but they have the added benefit of quantity and they are also the generators of ultimate value.

Connection strength between nodes: This incorporates strong and weak asso- 
ciations [11] [12]. Weak connections offer a great number of bridges linking additional networks, and numerous weak connections diffuse new information, occupying a leading role in information diffusion [13]-[19]. Strong associations bind user nodes together via close personal relationships. Virtually all of the social relationships happen between close friends who frequently know each other in real life [20]. Strong associations add to expanding human deportment on social media, online or in real life, and strong associations are more persuasive between individuals.

User effect: This involves deportment or abilities impacting individuals without noticeable compulsory measures or immediate orders.

User preference: This refers to if users are curious about the information they obtain.

User activity: User activity possesses high- and low-attribute values.

Limited user attention: This refers to the information captivating users.

Similarities between users: This involves the confluence of user features in terms of deportment, interest, activity, language, and other factors. Homogenous users are more frequently linked than divergent users.

User behavior: This incorporates three behaviors: forwarding, purchasing, and becoming information nodes. Forwarding involves the dispersal of information users obtain via their personal social media. Purchasing occurs in which the users are curious about the product endorsed by the information and then buy the product to create value, thereby becoming value nodes. Becoming information nodes describes users viewing information, but they do not act on it. For ease in performing later reasoning of the model, every variable was indicated by a corresponding symbol (Table 1).

\subsection{Establishment of the Bayesian Network Model of Information Diffusion}

A typical manner to generate a formation is via backward construction; it starts with a variable of interest (e.g., user deportment) for which we then attempt to discover its prior probability. If the probability is unclear due to its dependence on additional elements, then other elements will be included as the father nodes of the variable and then taken into the network. Finally, when we establish the formation, we will need to note that approximation could not be precluded.

We can set up a situation for the aforementioned variables in which a variable is dependent on other variables. There are numerous weaker effects as well as the connections among the noted variables. Nonetheless, if all of the variables were accounted for, the network would become too intricate; these types of networks are difficult to comprehend and mend, and the parameters are challenging to establish. Further, as Bayesian network reasoning is greatly reliant on connection function, adding the edges would likely make the utilization of the network very expensive. Figure 2 reveals the network arrangement of product information diffusion and its accompanying symbol portrayal. 
Overall, every variable in the model is connected with a CPD. This is utilized to indicate the dispersion of the value of this variable under the condition that each joint assignment of its father node is established. For nodes without father nodes, CPD is subject to an empty variable set. Therefore, CPD is altered into a marginal distribution, such as $P(T)$ and $P(N)$. The network structure of information diffusion forms the Bayesian network $B^{\text {difussion }}$ along with CPD.

In $B^{\text {difussion }}$, there are two kinds of unique variable nodes, one is the "S-Strength of Connection". Variable S is denoted as the multiplexer of CPD. That is to say, the a priori probability of the chosen variable is a duplicate of one of the values of its father nodes.

Packaged CPD variables are an additional kind of unique variable node, namely, "N-Near" and "I-Effect", and their priori probabilities were determined by other variables.

Table 1. Symbolic depiction, type, and value of variables in information diffusion networks.

\begin{tabular}{|c|c|c|c|}
\hline Name of variable & Symbol & Type & Value \\
\hline Cross diffusion & D-Cross diffusion & Binary variable & $\begin{array}{l}\left\{d^{0} \text { to the disadvantage of, }\right. \\
\left.d^{1} \text { to the advantage of }\right\}\end{array}$ \\
\hline Connection & C-Connection & Binary variable & $\left\{c^{0}\right.$ hard, $c^{1}$ easy $\}$ \\
\hline Theme & T-Themes & Integer variable & $\left\{t^{1}, \cdots, t^{k}\right\}$ \\
\hline $\begin{array}{l}\text { Emotion conveyed } \\
\text { by information }\end{array}$ & E-Emotion & Binary variable & $\left\{e^{0}\right.$ negative, $e^{1}$ positive $\}$ \\
\hline $\begin{array}{l}\text { Origin of } \\
\text { information }\end{array}$ & O-Origin & Boolean variable & $\left\{o^{0}\right.$ no, $o^{1}$ yes $\}$ \\
\hline $\begin{array}{l}\text { Resonance toward } \\
\text { the theme }\end{array}$ & R-Resonance & Binary variable & $\left\{r^{0}\right.$ not friends, $r^{1}$ friends $\}$ \\
\hline User state & U-Users & $\begin{array}{l}\text { Three-valued } \\
\text { variable }\end{array}$ & $\begin{array}{l}\left\{u^{0} \text { information node, }\right. \\
u^{1} \text { diffusion node, } u^{2} \\
\text { seed node }\}\end{array}$ \\
\hline $\begin{array}{l}\text { Connection strength } \\
\text { between nodes }\end{array}$ & $\begin{array}{l}\text { S-Strength of } \\
\text { connection }\end{array}$ & Binary variable & $\left\{s^{0} \quad\right.$ weak, $s^{1}$ strong $\}$ \\
\hline User effect & I-Effect & Binary variable & $\left\{i^{0} \quad\right.$ small, $i^{1} \quad$ big $\}$ \\
\hline User preference & F-Preference & Binary variable & $\begin{array}{l}\left\{f^{0} \text { disinterested, } f^{1}\right. \\
\text { interested }\}\end{array}$ \\
\hline User activity & A-Activity & Binary variable & $\left\{a^{0}\right.$ low, $a^{1}$ high $\}$ \\
\hline $\begin{array}{l}\text { Limited user } \\
\text { attention }\end{array}$ & L-Limited attention & Binary variable & $\begin{array}{l}\left\{l^{0} \text { not attracted, } l^{1}\right. \\
\text { attracted }\}\end{array}$ \\
\hline $\begin{array}{l}\text { Similarities between } \\
\text { users }\end{array}$ & N-Near & Binary variable & $\left\{n^{0}\right.$ low, $n^{1}$ high $\}$ \\
\hline User behavior & B-Behavior & Binary variable & $\begin{array}{l}\left\{b^{0} \text { forward, } b^{1} \text { purchase, }\right. \\
\left.b^{2} \text { information node }\right\}\end{array}$ \\
\hline
\end{tabular}



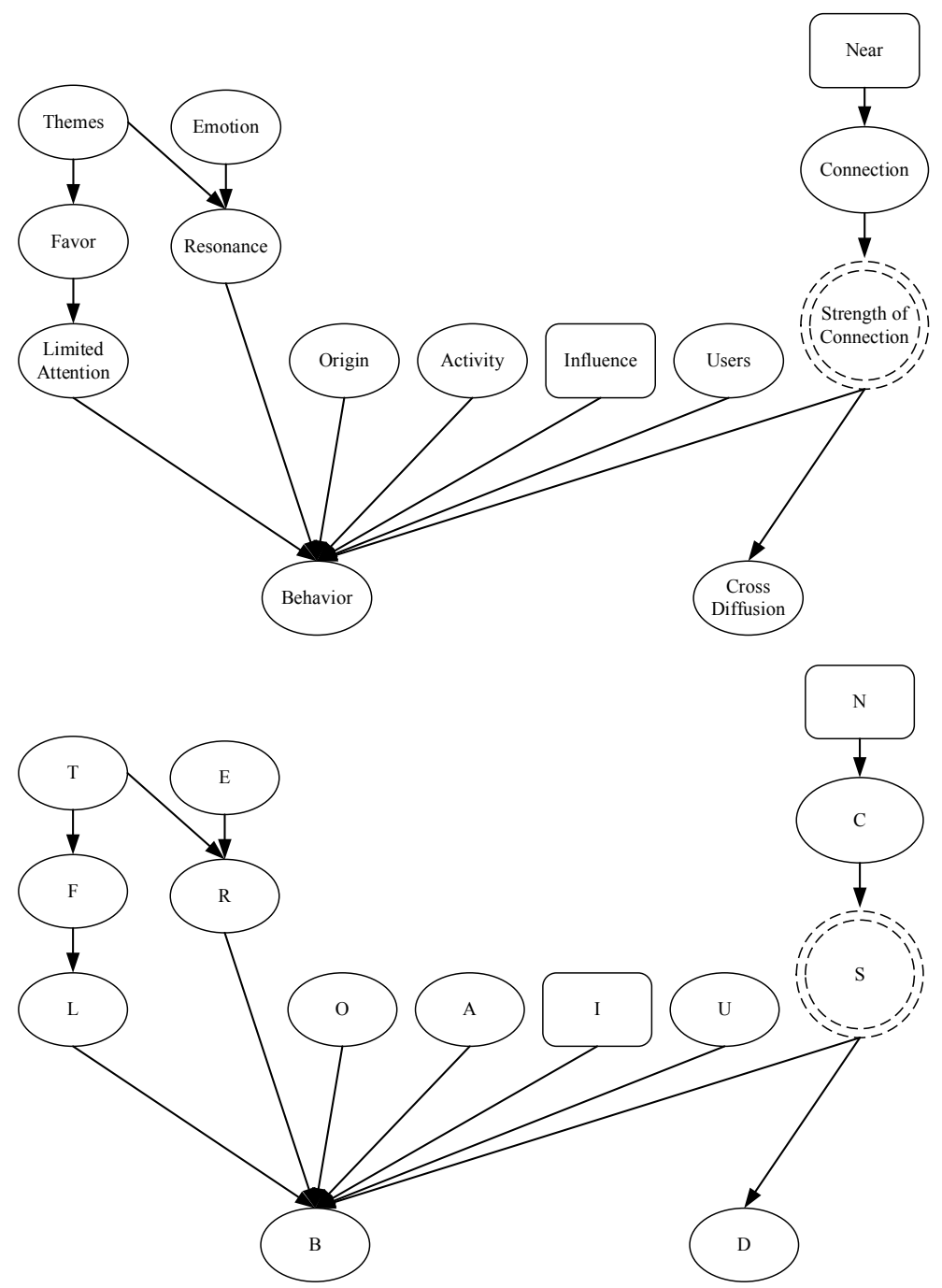

Figure 2. Network structure and symbolic depiction $G_{\text {diffusion }}$ of product information diffusion.

\section{Influence Reasoning Process}

Cooper [21] for the first time, conducted a formal analysis of the computational complexity of probability reasoning in the Bayesian network. Variants of the variable elimination algorithm were invented independently by multiple teams. An early variant came from the peeling algorithm proposed by Cannings et al. [22] [23], which is a systematic exposition of genetic lineage analysis.

The general problem of probability reasoning in the graph model was first solved by Kim and Pearl [24], who proposed a local message passing algorithm in the Bayesian network featuring the multiple tree structure. These views provoked the development of various algorithms that are more common, including a series of methods proposed by scholars, such as [25]-[30]; all of these methods end with the variable elimination algorithm. These methods were first described by Zhang and Poole [31] and then developed by Dechter, while Huang and Darwiche [32] provided some useful techniques for the effective implementation of 
such algorithms.

Following the idea of the multi-tree algorithm, Pearl presented a simple approach, namely generating multiple trees with clustering nodes, but the efficiency of the process is low. The sum-product message passing algorithm was developed by Shenoy and Shafer [33]. They described it with a very broad form, which, in addition to the probability graph model, also applies to many factorization models. The sum-product-division method was developed in a series of papers by Lauritzen and Spiegelhalter [34] and Jensen, Olesen, and Andersen [35]. Studies in this direction have also produced the theory that takes message passing operations as a re-parameterization implemented on the initial distribution. The sum-product-division algorithm described by Andersen, Olesen, and Jensen [36] formed the basis of the Bayesian network system, which then led to the extensive application of this method.

Influence reasoning used the clique tree data structure to pass influences or information between adjacent cliques. First, all of the influences were sent to the root clique to work out the marginal posterior probability of $P(B)$. On this basis, the root clique sent the information to the sub-cliques until all of the leaf nodes were used to estimate the marginal posterior probability of factors influencing consumer behavior choice and obtain the quantitative correlation between them.

\subsection{Mechanism of Influence Reasoning}

The influencing reasoning process includes the upward pass and the downward pass. When passing upward, a root node is selected to send all of the influences to the root clique, and when the process ends, the root node has all of the influences. Thus, the root node could now send appropriate information to its child node (mapped to $B^{\text {difussion }}$, that is, to its father node). The algorithm continues until the leaf node of the tree that is used to send information is no longer needed. The second stage is called the downward pass.

The two transfer factors of each edge can be calculated according to the following method. When a clique receives all of the influences sent by its downstream neighbors, it can send influences to the upstream clique. Assume $T$ as a clique tree; if $C_{i}$ receives information from all of the neighbors except for $C_{j}$, then the information passing from $C_{i}$ to $C_{j}$ is ready. When the message passing from $C_{i}$ to $C_{j}$ is ready, it can be calculated $\delta_{i \rightarrow j}\left(S_{i, j}\right)$ by multiplying its initial potential with information sent from all of its neighbors except for $C_{j}$, and the variables in $C_{i}-S_{i, j}$ are eliminated. The message passing process is as follows:

1) Initialization of the clique tree.

2) When there is $i, j$, that makes the transmission from $i$ to $j$ ready.

3) $\delta_{i \rightarrow j}\left(S_{i, j}\right) \leftarrow$ Message/Influence $(i, j)$.

4) Initialize each clique.

5) $\beta_{i}=\psi_{i} \cdot \prod_{k \in N b_{i}} \delta_{k \rightarrow i}$.

6) Return to $\left\{\beta_{i}\right\}$. 


\subsection{Reasoning of All Factors in the Product Information Diffusion Bayesian Network Model}

Figure 3 shows the upward pass of the clique tree algorithm with $C_{12}$ as the root clique in the information diffusion network and the downward pass on this basis. Based on influences from $C_{5}$ and its own initial potential energy, $C_{12}$ sends messages to its child node $C_{11}$. Once the child node of the root group receives the message, it will have all of the messages that need to be sent to its child node; for example, messages passed downward from $C_{11}$ to $C_{10}$. At the end of the process, all of the cliques in the tree can be calculated by multiplying the initial potential energy with each incoming message factor. The key is that the message factor used to calculate $\beta_{i}$ is exactly the same as the influencing factor in the upward pass algorithm with $C_{i}$ as the root node. Therefore, if we initialize each clique, according to the downward pass algorithm, $\beta_{i}$ can be expressed by the following equation:

$$
\beta_{i}\left(C_{i}\right)=\sum_{x-C_{i}} \underset{\Phi}{\tilde{P}}(x)
$$

At the end of the process, each clique contains the marginal probability of the variables within its scope. In the upward pass, the clique containing one specific variable could be selected to calculate the marginal probability of this variable and eliminate the redundant variables in the clique. The key point is that the results obtained by this process do not depend on the clique selected; that is, if the variables appear in two cliques, then their marginal probabilities must be consistent. The influence reasoning process is shown in Figure 3.

\section{Analysis and Verification of the Reasoning Process}

In the above algorithm, only when influences are sent from $C_{i}$ to $C_{j}$, and $X \in C_{i}$ and $X \notin C_{j}$, could the variable $X$ be eliminated. If $X$ is eliminated when the influences are sent from $C_{i}$ to $C_{j}$, then $X$ will not appear at the $C_{j}$ side of the edge $(i-j)$ in the tree. If $X$ appears in $C_{k}$, which is a specific clique at the $C_{j}$ side in the tree, $C_{j}$ will be on the path from $C_{i}$ to $C_{k}$. However, we know that $X$ appears simultaneously in $C_{i}$ and $C_{k}$, but it does not appear in $C_{j}$, thus it violates the running intersection property.

Based on this finding, a semantic interpretation is provided for the influences applied in the clique tree. Assume $(i-j)$ as one edge in the clique tree, $F_{\prec(i \rightarrow j)}$ denotes the set of factors at the $C_{i}$ side of the edge in the clique, and $V_{\prec(i \rightarrow j)}$ represents the set of variables at the $C_{i}$ side but not in the cut set. For example, in the clique tree shown in Figure 3,

$$
F_{\prec(3 \rightarrow 6)}=\{P(T), P(F \mid T), P(R \mid E, T), P(L \mid F), P(E), P(B \mid L, R, O, U, I, A, S)\}
$$

and $V_{\prec(3 \rightarrow 6)}=\{T, F, E, L\}$. Intuitively, the product of all of the factors in $F_{\prec(i \rightarrow j)}$ is passed between $C_{i}$ and $C_{j}$, which is the marginalization (summation of all of the other variables) on all of the variables in the cut set. 


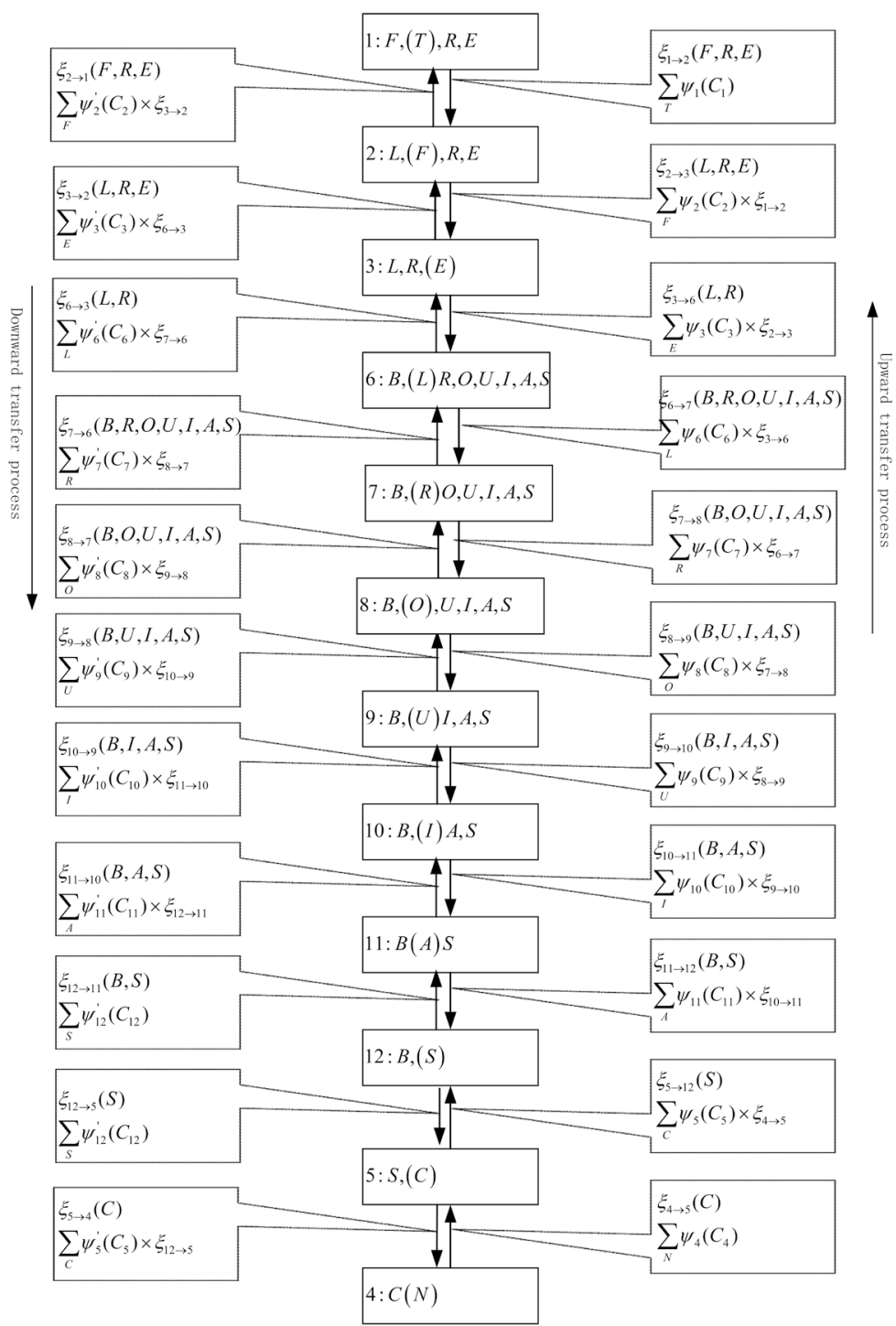

Figure 3. Upward and downward pass process of the information diffusion network.

In the above algorithm, $\delta_{i \rightarrow j}$ is an influence from $C_{i}$ to $C_{j}$, and $\delta_{i \rightarrow j}\left(S_{i, j}\right)=\sum_{V_{\langle(i \rightarrow j)}} \prod_{\phi \in F_{\langle(i \rightarrow j)}} \phi$. First of all, the basic situation was considered, where clique $C_{i}$ was the leaf node in the tree. In this context, it could be directly calculated by the operation on the clique, as shown in Figure 3. If clique $C_{i}$ is not a leaf node, then $\sum_{V_{\langle(i \rightarrow j)}} \prod_{\phi \in F_{\langle(i \rightarrow j)}} \phi$. If $i_{1}, \cdots, i_{m}$ is the neighbor clique of $C_{i}$ rather than $C_{j}$, and because $X$ will not appear at the $C_{j}$ side of the edge $(i-j)$ in the tree if $X$ is eliminated when the influences is sent from $C_{i}$ to $C_{j}$, $V_{\prec\left(i_{k} \rightarrow i\right)}$ has a disjoint union, $V_{\prec(i \rightarrow j)}$, where $k=1, \cdots, m$, and the variable $Y_{i}$ eliminates itself at $C_{i}$. Similarly, $F_{\prec(i \rightarrow j)}$ is the disjoint union of $F_{\prec\left(i_{k} \rightarrow i\right)}$, and $\psi_{i}$ is calculated per factor $F_{i}$. Thus, 


$$
\sum_{V_{\prec(i \rightarrow j)}} \prod_{\phi \in F_{\prec(i \rightarrow j)}} \phi=\sum_{Y_{i}} \sum_{V_{(i \rightarrow i)}} \cdots \sum_{V_{\left(i_{m} \rightarrow i\right)}}\left(\prod_{\phi \in F_{\prec\left(i_{1} \rightarrow i\right)}} \phi\right) \cdots\left(\prod_{\phi \in F_{\prec\left(i_{m} \rightarrow i\right)}} \phi\right)\left(\prod_{\phi \in F_{i}} \phi\right)
$$

As shown above, for each $k$, the variables in $V_{\prec\left(i_{k} \rightarrow i\right)}$ will not be found in any other factor. Therefore, based on the simple rule that the sum and the product of a factor are interchangeable, the following equation is obtained from the summation equation on $V_{\prec\left(i_{k} \rightarrow i\right)}$ in Equation (2):

$$
\sum_{Y_{i}}\left(\prod_{\phi \in F_{i}} \phi\right) \cdot \sum_{V_{\left(i_{1} \rightarrow i\right)}}\left(\prod_{\phi \in F_{\prec(i \mathfrak{i} \rightarrow i)}} \phi\right) \ldots \sum_{V_{\left(i_{m} \rightarrow i\right)}}\left(\prod_{\phi \in F_{\prec\left(i_{m} \rightarrow i\right)}} \phi\right)
$$

According to the inductive assumption and the definition of $\psi_{i}$, the above equation is equivalent to:

$$
\sum_{Y_{i}} \psi_{i} \cdot \delta_{i_{1} \rightarrow i} \cdots \delta_{i_{m} \rightarrow i}
$$

Equation (4) is the very operation to calculate the influencing factors in the upward pass algorithm and the message factors in the downward pass algorithm. Thus, the cut set can divide the graph into conditionally independent fragments, and such conditional independence enables messages on the cut set to completely sum up the information on one side of the clique tree, which is extremely important for the calculation of the information on the other side. Based on this analysis, we calculated the posterior probability of the variables in the selected root clique, and if $C_{r}$ is the root clique of the tree, then:

$$
\beta_{r}\left(C_{r}\right)=\sum_{x-C_{r}} \tilde{P}_{\Phi}(x)
$$

For the Bayesian network $B$, if $\Phi$ is composed by the CPDs in $B$ and it is simplified by the influencing factor $e$, then $\beta_{r}\left(C_{r}\right)=P_{B}\left(C_{r}, e\right)$. Moreover, the joint probability of this factor should be normalized as 1 before the marginal posterior probability of the variables on $C_{r}$ can be calculated.

\section{Verification of Influence Reasoning}

Assuming that the prior probabilities of all of the influencing factors are randomly generated, the upward pass reasoning was performed first to gain the marginal posterior probability of $P(B)$; on this basis, the downward pass reasoning was conducted to obtain the marginal posterior probability of each influencing factor; after 500 iterations the mean and standard deviation of each influencing factor were obtained, as shown in Table 2. Then, we can obtain the degree to which each influencing factor affects consumer behavior, and quantify the relationship between the influencing factors and user behavior.

\section{Conclusions and Future Research}

In this study, based on the product information diffusion in consumers' self-organized social networks, the factors affecting consumer behavior choice were explored during the product information diffusion process, and the main achievements and innovations are: 
Table 2. Marginal posterior probability, mean value, and standard deviation of influencing factors.

\begin{tabular}{|c|c|c|c|}
\hline Variable & $P(X)$ & mean & std \\
\hline \multirow{2}{*}{ T-Theme } & 0.3584 & 0.4703 & 0.3116 \\
\hline & 0.6416 & 0.5297 & 0.3116 \\
\hline \multirow{2}{*}{ E-Emotion } & 0.5687 & 0.5014 & 0.2647 \\
\hline & 0.4313 & 0.4986 & 0.2647 \\
\hline \multirow{2}{*}{ O-Origin } & 0.0466 & 0.5020 & 0.2397 \\
\hline & 0.9534 & 0.4980 & 0.2397 \\
\hline \multirow{2}{*}{ A-Activity } & 0.4773 & 0.5040 & 0.2353 \\
\hline & 0.5227 & 0.4960 & 0.2353 \\
\hline \multirow{2}{*}{ I-Influence } & 0.5808 & 0.4984 & 0.2381 \\
\hline & 0.4192 & 0.5016 & 0.2381 \\
\hline \multirow{3}{*}{ U-Users } & 0.0672 & 0.3370 & 0.1801 \\
\hline & 0.7697 & 0.3228 & 0.1827 \\
\hline & 0.1631 & 0.3401 & 0.1824 \\
\hline \multirow{2}{*}{ N-Near } & 0.6779 & 0.5004 & 0.2784 \\
\hline & 0.3221 & 0.4996 & 0.2784 \\
\hline \multirow{2}{*}{ F-Favor } & 0.2816 & 0.5115 & 0.2386 \\
\hline & 0.7184 & 0.4885 & 0.2386 \\
\hline \multirow{2}{*}{ L-Limited Attention } & 0.8481 & 0.4882 & 0.1976 \\
\hline & 0.1519 & 0.5118 & 0.1976 \\
\hline \multirow{2}{*}{ R-Resonance } & 0.1769 & 0.4085 & 0.2278 \\
\hline & 0.8231 & 0.5915 & 0.2278 \\
\hline \multirow{2}{*}{ C-Connection } & 0.2268 & 0.4996 & 0.2961 \\
\hline & 0.7732 & 0.5004 & 0.2961 \\
\hline \multirow{2}{*}{ S-Strength of Connection } & 0.6181 & 0.4938 & 0.1729 \\
\hline & 0.3819 & 0.5062 & 0.1729 \\
\hline
\end{tabular}

1) Multiattribute Analysis of Product Information Diffusion. Of the factors impacting the product information diffusion process, those with critical values were identified, and the causal link between them was evaluated. Rational ranges were designated to the binary, three-valued, and Boolean variables of those factors to more precisely evaluate the intricacy of the product information diffusion process.

2) Bayesian Model of Product Information Diffusion. A Bayesian network was implemented to depict information diffusion operations under the effects of multiple elements. It revealed how those factors had an impact on each other and on consumer deportment choice. It also demonstrated the process and construction generated by the product information diffusion network, thereby demonstrating the product information diffusion network in a more precise form.

3) Influence Reasoning in Product Information Diffusion. The marginal posterior probability of factors leading to the specific behaviors of users was estimated with a quantitative method so as to gain the quantitative association between user behavior and the influencing factors. 
Dynamic Bayesian network model of information diffusion considering the time factor would be proposed in the future research to analyze the associations between factors affecting the diffusion of product information among consumers and consumer behaviors.

\section{Acknowledgements}

This work is supported by Department of Education of Hebei Province [grant number QN2018259].

\section{Conflicts of Interest}

The authors declare no conflicts of interest regarding the publication of this paper.

\section{References}

[1] Kempe, D., Kleinberg, J. and Tardos, E. (2003) Maximizing the Spread of Influence through a Social Network. In: KDD2003: Proceedings of the Ninth ACM SIGKDD International Conference on Knowledge Discovery and Data Mining, ACM, New York, 137-146. https://doi.org/10.1145/956750.956769

[2] Clifford, P. and Sudbury, A. (1973) A Model for Spatial Conflict. Biometrika, 60, 581-588. https://doi.org/10.1093/biomet/60.3.581

[3] Chen, W., Collins, A., Cummings, R., et al. (2011) Influence Maximization in Social Networks When Negative Opinions May Emerge and Propagate. Proceedings of the Eleventh SIAM International Conference on Data Mining, Mesa, 28-30 April 2011, 379-390. https://doi.org/10.1137/1.9781611972818.33

[4] He, X., Song, G., Chen, W. and Jiang, Q. (2012) Influence Blocking Maximization in Social Networks under the Competitive Linear Threshold Model. SDM 2012, Anaheim, California, 2012, 463-474. https://doi.org/10.1137/1.9781611972825.40

[5] Li, Y., Chen, W., Wang, Y. and Zhang, Z.L. (2013) Influence Diffusion Dynamics and Influence Maximization in Social Networks with Friend and for Relationships. In: Proceedings of the Sixth ACM International Conference on Web Search and Data Mining, ACM, New York, 657-666. https://doi.org/10.1145/2433396.2433478

[6] Zhang, X., Zhu, J., Wang, Q., et al. (2013) Identifying Influential Nodes in Complex Networks with Community Structure. Knowledge-Based Systems, 42, 74-84. https://doi.org/10.1016/j.knosys.2013.01.017

[7] Chen, D., Lu, L., Shang, M.S., et al. (2012) Identifying Influential Nodes in Complex Networks. Physica A, 391, 1777-1787. https://doi.org/10.1016/i.physa.2011.09.017

[8] Kitsak, M., Gallos, L.K., Havlin, S., et al. (2010) Identification of Influential Spreaders in Complex Networks. Nature Physics, 6, 888-893.

https://doi.org/10.1038/nphys1746

[9] Kempe, D., Kleinberg, J. and Tardos, E. (2005) Influential Nodes in a Diffusion Model for Social Networks. Lecture Notes in Computer Science, 3580, 1127-1138. https://doi.org/10.1007/11523468_91

[10] Bae, J. and Kim, S. (2014) Identifying and Ranking Influential Spreaders in Complex Networks by Neighborhood Coreness. Physica A, 395, 549-559. https://doi.org/10.1016/j.physa.2013.10.047

[11] Granovetter, M.S. (1973) The Strength of Weak Ties. American Journal of Sociology, 78, 1360-1380. https://doi.org/10.1086/225469 
[12] Granovetter, M.S. (1983) The Strength of Weak Ties: A Network Theory Revisited. Sociological Theory, 1, 201-233. https://doi.org/10.2307/202051

[13] Montgomery, J.D. (1992) Job Search and Network Composition: Implications of the Strength of Weak Ties Hypothesis. American Sociological Review, 57, 586-596. https://doi.org/10.2307/2095914

[14] Kavanaugh, A.L., Reese, D.D., Carroll, J.M. and Rosson, M.B. (2005) Weak Ties in Networked Communities. The Information Society, 21, 119-131.

https://doi.org/10.1080/01972240590925320

[15] Harson, M.T. (1999) The Search-Transfer Problem: The Role of Weak Ties in Sharing Knowledge across Organization Subunits. Administrative Science Quarterly, 44, 82-111. https://doi.org/10.2307/2667032

[16] Friedkin, N.E. (1982) Information Flow through Strong and Weak Ties in Intraorganizational Social Networks. Social Networks, 3, 273-285.

https://doi.org/10.1016/0378-8733(82)90003-X

[17] Weimann, G. (1983) The Strength of Weak Conversational Ties in the Flow of Information and Influence. Social Networks, 5, 245-267. https://doi.org/10.1016/0378-8733(83)90027-8

[18] Centola, D. and Macy, M. (2007) Complex Contagions and the Weakness of Long Ties. American Journal of Sociology, 113, 702-734. https://doi.org/10.1086/521848

[19] Bakshy, E., Rosenn, I., Marlow, C. and Adamic, L. (2012) The Role of Social Networks in Information Diffusion. In: Proceedings of the 21 st International Conference on World Wide Web, ACM, New York, 519-528. https://doi.org/10.1145/2187836.2187907

[20] Pan, R.K. and Saramaki, J. (2012) The Strength of Strong Ties in Scientific Collaboration Networks. Europhysics Letters, 97, Article No. 18007. https://doi.org/10.1209/0295-5075/97/18007

[21] Cooper, G. (1990) Probabilistic Inference Using Belief Networks Is NP-Hard. Artificial Intelligence, 42, 393-405. https://doi.org/10.1016/0004-3702(90)90060-D

[22] Cannings, C., Thompson, E.A. and Skolnick, H.H. (1976) The Recursive Derivation of Likelihoods on Complex Pedigrees. Advances in Applied Probability, 8, 622-625. https://doi.org/10.2307/1425918

[23] Cannings, C., Thompson, E.A. and Skolnick, M.H. (1978) Probability Functions on Complex Pedigrees. Advances in Applied Probability, 10, 26-61. https://doi.org/10.2307/1426718

[24] Kim, J. and Pearl, J. (1983) A Computational Model for Combined Causal and Diagnostic Inference in Inference Systems. Proc. 7 th International Joint Conference on Artificial Intelligence, Vancouver, 1981, 190-193.

[25] Schachter, R.D. (1998) Bayes-Ball: The Rational Pastime. Proc. 14th Conference on Uncertainty in Artificial Intelligence (UAI), Madison, Wisconsin, 1998, 480-487.

[26] Shachter, R.D., DAmbrosio, B. and Del Favero, B.A. (1990) Symbolic Probabilistic Inference in Belief Networks. Proceedings of the 8 th National Conference on Artificial Intelligence. Boston, Massachusetts, 29 July-3 August 1990, 2, 126-131.

[27] Dechter, R. (1999) Bucket Elimination: A Unifying Framework for Inference. Artificial Intelligence, 113, 41-85. https://doi.org/10.1016/S0004-3702(99)00059-4

[28] Shafer, G. and Shenoy, P.P. (1990) Probability Propagation. Annals of Mathematics and Artificial Intelligence, 2, 327-352. https://doi.org/10.1007/BF01531015

[29] Dawid, A. (1992) Application of a General Propagation Algorithm for Probabilistic Expert System. Statistics and Computing, 2, 25-36. 
https://doi.org/10.1007/BF01890546

[30] Darwiche, A. (2003) A Differential Approach to Inference in Bayesian Networks. Journal of the ACM, 50, 280-305. https://doi.org/10.1145/765568.765570

[31] Zhang, N. and Poole, D. (1994) A Simple Approach to Bayesian Network Computations. Proceedings of the 10 th Biennial Canadian Artificial Intelligence Conference, Banff, Alberta, 1994, 171-178.

[32] Huang, C. and Darwiche, A. (1996) Inference in Belief Networks: A Procedural Guide. International Journal of Approximate Inference, 15, 225-263.

https://doi.org/10.1016/S0888-613X(96)00069-2

[33] Shenoy, P. and Shafer, G. (1990) Axioms for Probability and Belief-Function Propagation. Machine Intelligence and Pattern Recognition, 9, 169-198. https://doi.org/10.1016/B978-0-444-88650-7.50019-6

[34] Lauritzen, S.L. and Spiegelhalter, D.J. (1988) Local Computations with Probabilities on Graphical Structures and Their Application to Expert Systems. Journal of the Royal Statistical Society, Series B, 50, 157-224. https://doi.org/10.1111/j.2517-6161.1988.tb01721.x

[35] Jensen, F.V., Olesen, K.G. and Andersen, S.K. (1990) An Algebra of Bayesian Belief Universes for Knowledge-Based Systems. Networks, 20, 637-659.

https://doi.org/10.1002/net.3230200509

[36] Andersen, S., Olesen, K. and Jensen, F. (1989) HUGIN-a Shell for Building Bayesian Belief Universes for Expert Systems. Proceedings of 11 th International Joint Conference on Artificial Intelligence (IJCAI), Detroit, 1989, 1080-1085. 